\title{
Síndrome de Ehlers-D anlos con especial énfasis en el síndrome de hiperlaxitud articular
}

\author{
Jaime F Bravo \\ Ehlers-D anlos syndrome, \\ with special emphasis in the joint \\ hypermobility syndrome
}

There is an urgent need to increase the awareness on the Joint Hypermobility Syndrome (JHS). This is a congenital and prevalent emergent condition that is frequently undiagnosed and that causes significant health problems. Besides recurrent musculoskeletal problems and signs and symptoms derived from tissue fragility, adolescents and young adults may develop osteoporosis, early osteoarthritis or dysautonomia, that are common in the disease, and deteriorate quality of life. Many JHS patients have signs and symptoms suggestive of fibromyalgia and are usually misdiagnosed. Physicians should be able to differentiate the less severe form of JHS from the Vascular Ehlers-Danlos Syndrome, to diagnose it before the appearance of serious complications and even death. The study of these diseases is a promising area for genomic and rheumatologic research (Rev Méd Chile 2009; 137: 1488-97).

(Key words: Ehlers-Danlos syndrome; Fibromyalgia; Joint instability)

Recibido el 23 de marzo, 2008. Aprobado el 19 de enero, 2009.

Departamento de Reumatología, Departamento de Medicina Occidente, Facultad de Medicina, Universidad de Chile, Hospital San Juan de Dios. Santiago de Chile.

$\mathrm{E}^{\mathrm{n}}$ n general la hipermovilidad articular es considerada como una habilidad o "un juego de niños", en vez de un problema potencialmente serio. Muchas personas tienen articulaciones hipermovibles, sin síntomas, hipermovilidad articular $(\mathrm{HA})^{1}$. Su prevalencia es difícil de establecer debido a que se han usado diferentes criterios diagnósticos y porque su frecuencia varía dependiendo de la raza, edad y sexo. Existiría en 10\% a $15 \%$ de la población occidental ${ }^{2}$. Su frecuencia es

Correspondencia a: Dr. Jaime F Bravo. Departamento de Reumatología. Hospital San Juan de Dios. Chacabuco 419. Santiago. Chile. Fono: 2-5708505. Fax: 2-335-9263.

E mail: jaime.bravos@gmail.com mayor en los asiáticos y negros, y en éstos, más que en los caucásicos. Es más frecuente en mujeres y niños. En la mayoría de los casos el compromiso es pauciarticular, lo que dificulta el diagnóstico ${ }^{3}$.

Cuando la HA presenta síntomas, constituye el síndrome de hiperlaxitud articular (SHA), el que no sólo afecta articulaciones y tendones, sino también algunos órganos, debido a debilidad de los tejidos. El score de Beighton (scB), es un puntaje que se obtiene sumando un grupo determinado de articulaciones hipermovibles. El puntaje máximo es de 9 sobre 9 (9/9) y generalmente en adultos se usa un punto de corte de 4 sobre 9 (4/9) para considerarlo positivo. Es el método más frecuentemente usado internacionalmente para evaluar la hiperlaxitud articular ${ }^{4}$. Considera sólo 
algunas articulaciones y no sirve por sí solo para el diagnóstico del SHA. El criterio de Brighton (CB) agrega al puntaje obtenido en el scB, la evaluación de otros tejidos alterados y confirma el diagnóstico de SHA cuando es positivo en ausencia de exclusiones y ha sido validado sólo para pacientes de 16 años o más 5 .

El tejido conectivo es la estructura intercelular que da resistencia a los tejidos. Está compuesto por varias proteínas, incluyendo colágenos, proteoglicanos y glicoproteínas. Algunas enfermedades hereditarias del tejido conectivo (EHTC) se consignan en la Tabla $1^{6}$.

\section{SíndRome De Ehlers-Danlos vascular (SEDV)}

Se presenta entre 1 y 2 por 100.000 habitantes. Tiene herencia autosómica dominante ${ }^{7}$. La mitad de los pacientes son portadores de la mutación COL3A1, el resto desarrolla la enfermedad debido a "una mutación de novo".

Se caracteriza por hematomas recurrentes y tendencia a rupturas arteriales y de órganos, por lo que es de pronóstico grave. Para el diagnóstico del SEDV se usa el criterio de Villefranche ${ }^{6}$ (Tabla 2). Pope demostró que esta condición se debe a la ausencia o disminución del colágeno tipo III, que

\section{Tabla 1. Clasificación de las alteraciones hereditarias del tejido conectivo ${ }^{6}$}

A) Síndrome de Ehlers-Danlos (SED):

Los 10 tipos iniciales se han reducido a 6:

SED clásico (SEDC)

SED hipermovible *

SED vascular (SEDV)

SED de tipo cifoescoliosis

SED de tipo artrocalasia

SED de tipo dermatoparaxis

B) Sindrome de Marfán (SMF)

C) Osteogénesis imperfecta (OI)
Antiguamente llamado SED tipo I-II Antiguamente llamado SED tipo III Antiguamente llamado SED tipo IV Antiguamente llamado SED tipo VI Antiguamente incluido en el SED tipo VII Antiguamente incluido en el SED tipo VII

Nota: SMF, OI y SED, no están incluidos en esta revisión excepto por el SHA y el SEDV.

*Para la mayoría de los autores sería lo mismo que el síndrome de hiperlaxitud articular (SHA).

Tabla 2. Criterio diagnóstico del síndrome de Ehlers-D anlos vascular (SEDV) $)^{9,10}$

\begin{tabular}{|ll|}
\hline Signos mayores: & Signos menores: \\
\hline - Piel delgada y transparente & - Acrogeria (manos avejentadas) \\
(se ven las venas) & - Hipermovilidad de pequeñas articulaciones \\
- Aneurismas, disección arterial o & de los dedos \\
ruptura arterial, intestinal o uterina & - Ruptura de tendones o músculos \\
- Hematomas espontáneos, frecuentes & - Pie equino varo \\
- Cara con características típicas & - Várices precoces \\
(triangular, ojos hundidos, & - Fístulas arteriovenosas \\
labio superior delgado, falta de tejido & - Neumotórax espontáneo \\
adiposo de la cara) & - Recesión de las encías \\
& - Historia familiar de muerte súbita de algún \\
& familiar cercano joven, sin causa aparente \\
\hline
\end{tabular}

Se requieren dos signos mayores para el diagnóstico clínico. Los signos menores ayudan al diagnóstico. Para confirmar el diagnóstico son necesarios exámenes bioquímicos o genéticos. 
es el componente esencial de órganos distensibles como arterias, intestino, útero y pulmones, resultando en fragilidad de dichos órganos e incluso ruptura a temprana edad ${ }^{8}$. Es necesario que los médicos identifiquen pacientes y familias con SEDV y síndrome de Marfán (SMF), debido a que ellos pueden tener complicaciones graves, a veces fatales. Estudios invasivos y procedimientos quirúrgicos pueden ser riesgosos en estos enfermos ${ }^{9}$. Por lo general los pacientes con SEDV sólo tienen hiperlaxitud de los dedos, por lo que si existe hiperlaxitud generalizada probablemente se trata de SHA y no de SEDV. Es importante saber que los problemas más serios debidos al SEDV y al SMF, a veces fatales, $25 \%$ de ellos aparecen antes de los 20 y $80 \%$ antes de los 40 años de edad 6 . Reconocer la facies típica del SEDV facilita el diagnóstico $^{7-10}$ (Figura 1, Tabla 2). Éste se puede confirmar con exámenes bioquímicos o por estudios genético-moleculares ${ }^{10}$. Estos exámenes confirmatorios son caros y sólo se hacen en el extranjero.

\section{SíNDROME DE HIPERMOVILIDAD ARTICULAR (SHA)}

Es menos grave que el SEDV, aunque puede presentar complicaciones músculo-esqueléticas y de otros órganos. Se caracteriza por alteraciones de la piel, artralgias, mialgias, crujido de las articulaciones, subluxaciones y tendinitis recurrentes y dolor de espalda. El dolor del SHA se puede hacer crónico e incapacitante y a veces se asocia a fatiga crónica. Hay tendencia a artrosis y osteopo- rosis precoz. Su frecuencia es muy alta en clínicas reumatológicas, en las que se le ha buscado con esmero. En España, Guma lo encontró en $25 \%{ }^{11}$ y en Londres, Grahame en $45 \% 12,13$. Hay datos que indican que su prevalencia sería igualmente alta en la población chilena ${ }^{14,15}$. Según Grahame, el SHA es probablemente la causa más frecuente de dolor en pacientes reumatológicos $\mathrm{y}$, a pesar de esto, es raramente diagnosticado ${ }^{13,16}$. Un estudio en reumatólogos en Inglaterra, mostró una alta tasa de desconocimiento de esta enfermedad ${ }^{17}$. Son múltiples las razones de esta falta de interés mundial: se diagnostican problemas puntuales como tendinitis y bursitis. Si se reconoce la hiperlaxitud, no se aprecia su significado, ni sus posibles complicaciones. Su alta frecuencia, la falta de conocimiento de que puede causar daño a múltiples órganos, el hecho que el cuadro clínico no sea dramático, no haya signos inflamatorios y no existan exámenes de laboratorio ni signos radiológicos específicos y que existe la sensación de que el tratamiento no es efectivo, hace que los médicos no se interesen por este diagnóstico.

Child describió en pacientes con SHA, hace años, una alteración en la proporción del colágeno tipo I con el tipo III, semejante a lo observado para la osteogénesis imperfecta y la microscopia electrónica mostró fibras colágenas de menor diámetro ${ }^{18}$. Hermanns-Le (2007) encontró que las fibrillas de colágeno eran de un diámetro variable y el espacio ínterfibrilar presentaba abundante material gránulo-filamentoso. Las fibras elásticas presentaban anormalidades en $76 \%$ : fragmentación, microcalcificaciones e inclusiones de densi-

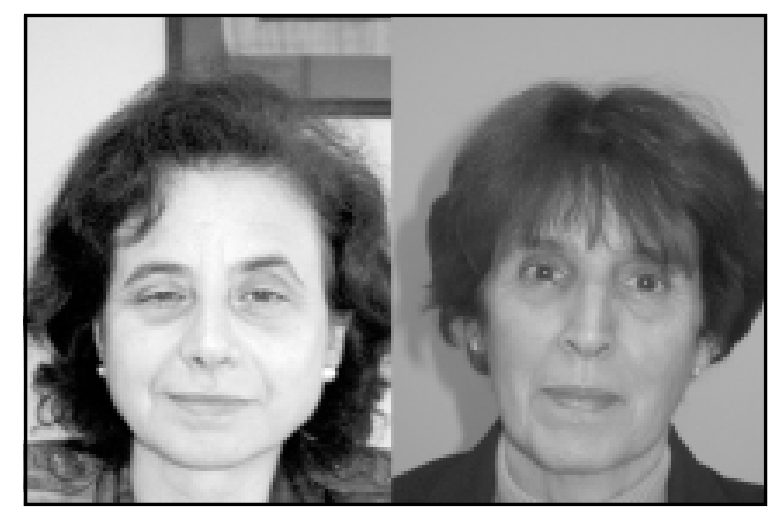

Figura 1. Facies típica de Ehlers-Danlos vascular (SEDV) ${ }^{7-10}$. (Fotografías con permiso expreso de los pacientes). 
dad electrónica aumentada en la matriz de la elastina, semejante a lo que se ve en la elastosis solar. Además grandes glóbulos de ácido hialurónico de forma estrellada y acumulaciones de depósitos gránulo-filamentosos en la matriz intersticial $^{19}$. A pesar de esto, todavía la alteración bioquímica precisa y el defecto genético en el SHA no son bien conocidos. Estudios recientes indican un probable rol de defectos del gen de Tenascina- $\mathrm{X}^{20}$. Tiene herencia autosómica dominante, al igual que la mayoría de las EHTC.

\section{SíNTOMAS Y SIGNOS}

El SHA tiene compromiso sistémico debido a que el colágeno está presente en todos los tejidos.

I. Síntomas músculo-esqueléticos:

A. Características generales:

- Piel suave, laxa, pálida, transparenta las venas, venas prominentes, mala cicatrización (cicatrices papiráceas, queloi- des), telangectasias, lívido reticularis. La laxitud de la piel se estudia en el dorso de la mano.

- Hematomas recurrentes. Confusión con maltrato infantil y con la enfermedad de von Willebrand 21 .

- Artralgias o mialgias por más de 3 meses.

- Dolor crónico difuso, poco anatómico, semejante a la fibromialgia (FM). Dolor en zonas de entesitis, semejantes a los "puntos dolorosos".

- Dolor de espalda, hiperlordosis lumbar, discopatías o hernia del núcleo pulposo, a temprana edad 5 .

- Historia de tendinitis, bursitis o lesiones deportivas recurrentes.

- Hiperlaxitud articular de una o más articulaciones.

- Crujidos articulares importantes.

- Posibilidad de extender el dedo meñique a $90^{\circ}$ o más; capacidad de extender los dedos "mano en forma de ave

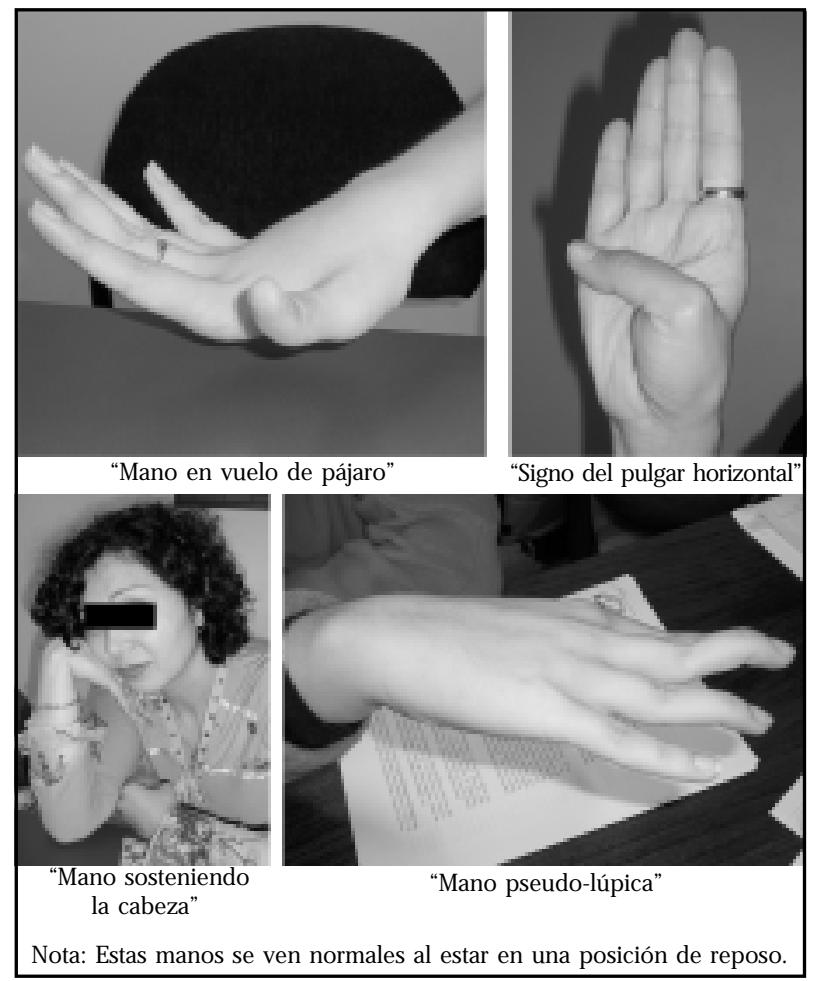

Figura 2. Signos de hipermovilidad en el SHA ${ }^{15}$. 
volando" o hacer "el signo del pulgar horizontal" o el signo de "afirmarse la cabeza con la mano"15 (Figura 2).

- Subluxaciones articulares, incluyendo problemas de la articulación temporomandibular.

- Artrosis precoz en personas jóvenes, artrosis erosiva y artrosis de evolución rápida. Ocasionalmente asociada a condrocalcinosis ${ }^{22,23}$.

- DMO baja u osteoporosis (O) en hombres y mujeres jóvenes, sin causa aparente. En pacientes con SHA, en la Unidad de Reumatología de adultos, encontramos osteoporosis en $22 \%$ y, lo que es más importante, existió en los menores de 30 años en 19\% en ambos $\operatorname{sexos}^{15}$. Es interesante notar que no hemos visto fracturas patológicas en estos enfermos jóvenes, probablemente porque tienen menor tendencia a caerse.

- Hallux valgus, en jóvenes.

- Hábito marfanoide (HMF): Jóvenes altos y delgados, con extremidades largas, aracnodactilia, a veces con pectum excavatum o pectum carinatus o con costillas prominentes ${ }^{5}$.

- En niños: Retardo en comenzar a caminar, "dolores del crecimiento", falta de concentración, torpeza en los movimientos, dislocaciones o subluxaciones recurrentes. Contorsiones del cuerpo ("niño de goma") o "actos malabares" con las manos.

B. Malformaciones congénitas asociadas:

Escoliosis en la infancia, displasia de cade$\mathrm{ra}$, rodillas rotadas, genu recurvatum, alteraciones de los pies (pie plano, laxo, cavo, pie egipcio, hallux valgus en jóvenes, sindactilia parcial), espina bífida oculta, espondilolistesis, pectus excavatum o carinatum y costillas prominentes.

II. Síntomas extraesqueléticos:

A. Alteraciones debidas a tejidos débiles:

- Escleras celestes, miopía, estrabismo.

- Acrocianosis, arritmias y PVM. El fenómeno de Raynaud en Chile, en el SHA parece ser muy poco frecuente $(2 \%)$, sólo 5 en 230 enfermos $^{15}$, comparado con esclerodermia $90 \%$ y LES $60 \% 24$. Raynaud en AR en otros países $17,2 \%{ }^{25}$. El fenómeno de Raynaud en países occidentales se describe en $4 \%$ y $15 \%$ de la población general ${ }^{26}$.

- Hernias, venas varicosas en gente joven, hemorroides, varicocele, prolapso vaginal o rectal, prolapso de la válvula mitral, quistes de todos tipos (Ganglion y quiste de Baker).

- Abortos, infertilidad, ruptura prematura de membranas, desgarros vaginales y hemorragias en el parto.

- Neumotórax espontáneo: Es más frecuente en el SMF, pero también se puede ver en el SHA de tipo marfanoide y también en el EDSV ${ }^{9,10}$.

- Constipación severa, incluso megacolon, reflujo, colon irritable y diverticulosis. Hipermovilidad de la lengua.

B. Síntomas neurofisiológicos:

a) Alteración del sistema nervioso autónomo (SNA):

1. Disautonomía (Dis). Es una disfunción autonómica caracterizada por fatiga crónica, mareos y síncopes ocasionales $^{15,27-29}$ (Tabla 3). Se presentó en $64 \%$ de las mujeres y en $40 \%$ de los hombres menores de 30 años, en

Tabla 3. Síntomas y signos de disautonomía ${ }^{15,27}$

- Hipotensión o hipotensión ortostática

- Cansancio, fatiga crónica y somnolencia

- Mareos e incluso síncope

- Intolerancia al frío

- Sudoración de manos y pies

- Acrocianosis, no solamente con el frío, sino también con dependencia e inactividad de las manos 
nuestro estudio ${ }^{15}$. Gazit ha notado diferencia estadística entre los hiperlaxos con hipotensión ortostática (22\%) y taquicardia postural ortostática (15\%) comparados con controles $(9,5 \%)^{29}$. Además $48 \%$ mostró intolerancia ortostática no catalogada. La Dis se debe a un desbalance del SNA y disminución del tono de las venas en las extremidades inferiores, secundario a la alteración del colágeno ${ }^{28}$.

2. Xeroftalmia y xerostomía ${ }^{15,28}$.

3. Colon irritable.

b) Percepción exagerada del dolor, con mala respuesta a los analgésicos loca$\operatorname{les}^{30}$ y frecuente existencia de dolor crónico, a veces invalidante.

c) Alteración de la propiocepción, con alteración del balance y coordinación ${ }^{31}$.

C. Síntomas neuropsiquiátricos:

La depresión, ansiedad, crisis de pánico y fobias se pueden heredar junto con el $\mathrm{SHA}^{32}$. Puede existir intranquilidad, cefaleas, jaquecas, piernas nerviosas, calambres, mala memoria, falta de concentración, desorientación y falta de motivación.

D. Otras características:

- Facies típica de SHA ${ }^{15}$ (Figura 3, Tabla 4).

\section{EXÁMENES COMPLEMENTARIOS}

No hay exámenes específicos de laboratorio para confirmar el diagnóstico clínico, sin embargo, es necesario pedir exámenes de rutina, exámenes inmunológicos y radiografías, para excluir otras enfermedades. El ecocardiograma es útil para detectar la dilatación aórtica y el PVM. El Tilt test sirve para confirmar el diagnóstico de Dis. Recomendamos hacer densitometrías óseas a todos los pacientes con SHA ${ }^{14,15,33}$.

\section{DiAgNóstico}

El diagnóstico es clínico y se confirma usando el criterio de Brighton ${ }^{5}$. Con práctica es fácil identificar a los pacientes que tienen la facies típica de SHA $^{15}$ (Figura 3, Tabla 4). Ayuda el saber que a algún familiar ya se le ha diagnosticado esta enfermedad. Pobre respuesta a los analgésicos inyectables locales ${ }^{30}$ y la presencia de alteraciones de la propiocepción ${ }^{31}$, deben alertar al diagnóstico del SHA.

Se debe sospechar especialmente en niñas adolescentes, en caso de disautonomia, caracterizada por aparecer como cansadas, somnolientas con intolerancia al frío, con mareos, con pocos deseos de hacer vida social y a veces con presíncope o desmayos, asociada a hipotensión arterial. Si sospechamos el SHA, debemos evaluar la hipermovilidad articular usando el scB y examinar la piel y otros tejidos débiles usando el $\mathrm{CB}^{5}$. El SEDV se puede sospechar clínicamente usando el criterio de Villefranche ${ }^{6}$, pero se puede confirmar con estudios bioquímicos y genéticos ${ }^{10}$. El SMF se diagnostica clínicamente usando el criterio de Ghent ${ }^{34}$.

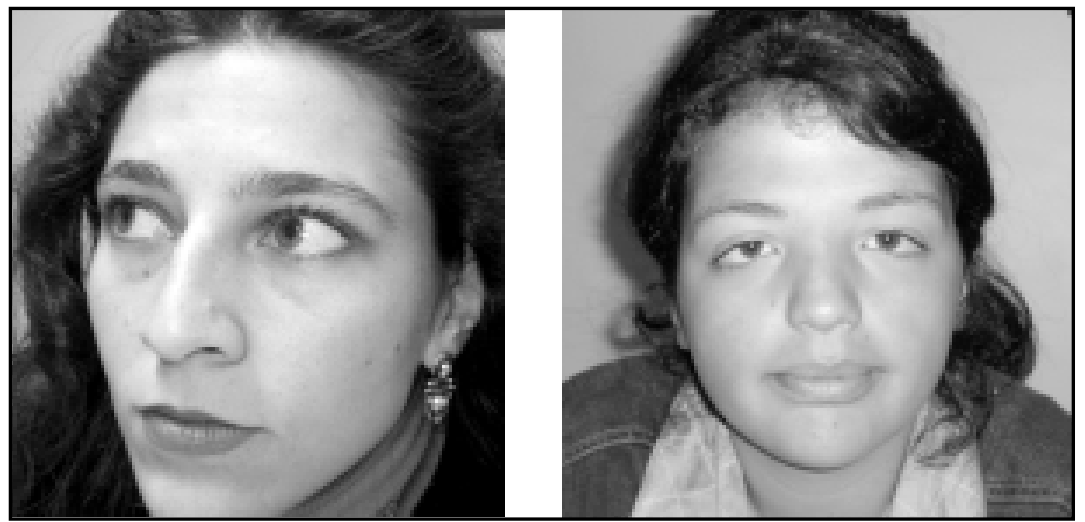

Figura 3. Facies típica del síndrome de hiperlaxitud articular (SHA) ${ }^{15}$. (Fotografías con permiso expreso de los pacientes). 


\section{Tabla 4. Facies típica del síndrome de hiperlaxitud articular (SH A) ${ }^{15}$}

- Cara triangular (mandíbula aguzada)

- Escleras celestes, más notorias en mujeres

- Orejas atípicas: prominentes "aladas"; pequeñas, redondas y sin lóbulo; lóbulo pegado a la cara. Diferentes formas: arriñonadas, orejas "Dumbo", orejas puntiagudas como el Sr. Spock, orejas blandas, orejas con hélix doblado; en forma de signo de interrogación; orejas operadas

- Nariz atípica: nódulo entre el hueso y el cartílago; tabique nasal desviado; nariz operada

- Hendidura palpebral antimongólica

\section{EL DIAGNÓSTICO DIFERENCIAL}

Se plantea con las otras EHTC, como el SEDV, SMF y OI. Es necesario diferenciar la facies típica del SHA ${ }^{15}$ (Figura 3, Tabla 4), de la facies típica del SEDV ${ }^{10}$ (Figura 1). El diagnóstico diferencial con casos tempranos de artritis como AR y LES puede ser difícil, pero una diferencia importante es que la sinovitis no es parte del SHA ${ }^{15,35}$. El SHA puede aparecer asociado a artritis, situación que hemos encontrado en $15 \%$ de los pacientes con $\mathrm{SHA}^{25}$. A veces se hacen diagnósticos erróneos, como en el caso de niñas con artralgias debidas a la hipermovilidad y a las que se diagnostica LES, especialmente si los anticuerpos antinucleares están en valores límites. Algunos pacientes con SHA pueden tener manos que semejan a las manos del LES ("mano pseudo-lúpica") (Figura 2).

El diagnostico diferencial con la FM presenta problemas si no se está familiarizado con los síntomas y signos del SHA. La presencia de fatiga crónica y el hecho que los dolores recurrentes de estos enfermos son en los sitios de entesis, precisamente en las mismas zonas de los "puntos dolorosos" de la FM nos hace pensar que es posible que la FM se asocie o sea parte del SHA. Gedalia encontró una asociación estadísticamente significativa entre hipermovilidad y FM en escolares $^{36}$ y Barron hizo notar que el síndrome de fatiga crónica era 3,5 veces más frecuente en adolescentes hipermóviles comparados con normales $^{37}$. Acasuso-Díaz encontró una clara asociación entre hipermovilidad y FM, en niños y adultos $^{38}$. Es importante señalar que los niños tienen problemas similares a los adultos y por lo general no son diagnosticados ${ }^{39}$.
También es necesario considerar el SHA en el diagnóstico diferencial de las artritis semegativas. A veces se piensa en Sjögren debido a la presencia de xeroftalmia y xerostomía, las que pueden existir en el SHA, debido a la alteración del SNA ${ }^{15,28}$.

\section{TRATAMIENTO}

No hay tratamiento curativo del SHA, por lo que el tratamiento es sintomático. El ingerir colágeno no ayuda. Es posible que en el futuro la terapia génica, sea una solución.

Medidas preventivas. Evitando la sobreextensión de las articulaciones es posible prevenir el dolor y la osteoartritis. Los ejercicios de estiramiento (stretching) son beneficiosos. Se recomienda hacer yoga, pilates, tai chi, natación y bicicleta. Son muy útiles los ejercicios destinados a fortalecer tendones y músculos ${ }^{40}$. Hay que evitar deportes y "hobbies" que producen dolor, tendinitis o subluxaciones en forma recurrente. Los deportes de contacto no son recomendables. A veces es necesario usar vendajes o férulas. Una buena medida es tomar períodos cortos de descanso y no mantener la misma posición corporal por horas. El conocer los problemas de la hiperlaxitud articular permite dar consejos sobre opciones para elegir una carrera profesional, instrumento musical, deporte o hobby.

Tratamiento de la fase aguda. Es necesario el reposo de la articulación afectada. Por lo general se requiere aplicación de calor o frío y a veces el uso de férulas. Son útiles los masajes, ultrasonido 
y ultratermia. Es necesario evitar la actividad repetitiva que produjo la lesión. Se recomienda paracetamol o antiinflamatorios por períodos cortos. Los relajantes musculares son de ayuda y las infiltraciones con esteroides están indicadas en las bursitis y tendinitis.

Tratamiento de la fase crónica. El reumatólogo es el especialista más indicado para tratar estos pacientes por su conocimiento de las EHTC, ya que es necesario ser capaz de diferenciar el SHA de las otras formas más graves como el SEDV, SMF y la OI. A pesar de que el dolor es el principal síntoma del SHA, los antiinflamatorios son de efecto limitado y es preferible usar analgésicos.

Kinesiterapia. La terapia ocupacional es muy útil en caso de ser efectuada por un terapeuta con conocimientos sobre la hipermovilidad articular, de lo contrario los resultados no son buenos. El principal logro es restablecer el rango de movilidad normal de la articulación, corregir la disfunción del movimiento, mejorando la estabilidad articular y la condición física general ${ }^{40}$. Los ejercicios deben fortalecer los músculos y tendones. Ocasionalmente es necesario inmovilizar alguna articulación, pero solamente durante el episodio agudo. La acupuntura y el TENS suelen ayudar y ocasionalmente puede ser necesario un tratamiento en una clínica de control del dolor. Existe un excelente libro sobre terapia física de los pacientes hipermovibles, publicado por Keer y Grahame ${ }^{40}$.

Medicamentos. Nosotros recomendamos a estos enfermos tomar de 0,4 a $1 \mathrm{mg}$ de ácido fólico (AF) diarios en forma permanente ${ }^{15}$, ya que hay estudios que demuestran que la deficiencia de $\mathrm{AF}$ altera los enlaces del colágeno, produciendo debilidad de los tejidos ${ }^{41}$. Para calambres es útil el magnesio y en caso de fragilidad capilar, además de evitar contusiones, es necesario indicar vitamina $\mathrm{C}$ o un polivitamínico. Es importante detectar y tratar precozmente la osteoartritis. El uso de glucosamina sola ${ }^{42}$ o con condroitin sulfato ${ }^{43}$, para prevenir la artrosis, es sugerido por algunos y parece ser útil, pero requiere de estudios que lo confirmen. El tratamiento de la $\mathrm{O}$ debe iniciarse lo antes posible de la forma usual.

Tratamiento de la disautonomía. Se trata con medidas generales, tomar 2 a 3 litros de líquidos al día, aumentar la ingesta de sal (excepto contraindicaciones), usar medias elásticas hasta la rodilla y reposar después de almuerzo. Hay que evitar la inactividad, estar de pie sin moverse y caminar despacio. De existir anemia o deshidratación, es necesario corregirlas. Estas medidas generales asociadas a medicamentos para elevar la presión arterial, tales como la etilefrina, midodrina y la fluodrocortisona, pueden mejorar significativamente la calidad de vida del paciente ${ }^{28}$.

Tratamiento en equipo. En ocasiones es necesaria la opinión de un cardiólogo, ginecólogo u otro especialista, incluyendo psicólogos, psiquiatras, fisiatras, kinesiólogos y terapeutas ocupacionales. Los grupos de soporte son de ayuda considerable, tanto para los enfermos como para los familiares. Ocasionalmente se requiere cirugía, como en el caso de subluxaciones recurrentes, pero es necesario recordar la mayor tendencia de hemorragias, infecciones y mala cicatrización en estos enfermos.

En conclusión es necesario que padres, profesores, entrenadores, terapeutas físicos, médicos y psicólogos conozcan la alta frecuencia y complicaciones asociadas a la hipermovilidad articular. También es importante informar a la opinión pública, a fin de aumentar la alerta diagnóstica de esta condición poco conocida, que parece ser la causa de las lesiones recurrentes de niños y deportistas chilenos.

\section{Agradecimientos}

Al bioquímico, Dr. Carlos Wolff y a la Dra. Cecilia Rojas por la lectura crítica de este manuscrito y al Sr. Hernán Isotta por su ayuda invaluable en la recopilación de los datos de los enfermos. 


\section{REFERENCIAS}

1. Mishra MB, Ryan $P$, Atkinson $P$, Taylor $H$, Bell J, CALVER D ET AL. Extra-articular features of benign joint hypermobility syndrome. Br J Rheumtol 1996; 35: 861-6.

2. GRAHAME R. Joint hypermobility and genetic collagen disorders: are they related? Arch Dis Child 1999; 80: 188-91.

3. Larsson LG, Baum J, Mudholkar GS, Srivastava DK. Hypermobility prevalence and features in Swedish population. Br J Rheumatol 1993; 32: 116-9.

4. Beighton PH, Solomon L, Soskolne CL. Articular mobility in an African population. Ann Rheum Dis 1973; 32: 413-8.

5. Grahame R, Bird HA, Child A, Dolan L, EdwardsFOWLER A, FERRELL WR ET AL. The revised (Brighton 1998) criteria for the diagnosis of the benign joint hypermobility syndrome. J Rheumatol 2000; 27: 1777-9.

6. Beighton P, Depaepe A, Steinmann B, Tsipouras P, WENSTRUP RJ. Ehlers-Danlos syndromes: revised nosology, Villefranche, 1977. Am J Med Gen 1998; 77: 31-7.

7. Pope FM, Martin GR, Lichtenstein JR, Penttinen R, Gerson B, Rowe DW, McKusic VA. Patients with Ehlers-Danlos type IV lack type III collagen. Proc Nat Acad Sci USA 1975; 72: 1314-6.

8. BRAVO JF. Precauciones y posibles complicaciones quirúrgicas de las alteraciones hereditarias de la fibra colágena (AHFC). Rev Chil Cirugía 2005; 57: 516-22.

9. Bravo JF. Síndrome de Ehlers-Danlos vascular (SEDV), antes llamado SED tipo IV. Rev Chil Reumatol 2008; 24: 6-11.

10. Pepin M, Schwarze U, Superti-Furga A, Byers PH. Clinical and genetic features of Ehlers-Danlos syndrome type IV, the vascular type. N Engl J Med 2000; 342: 673-80.

11. Guma M, Olivé A, Holgado S, Casado E, Roca J, ForCADA J ET AL. Una estimación de la laxitud articular en la consulta externa. Rev Esp Reumatol 2001; 28: 298-300.

12. GraHAME R, HaKim AJ. Joint hypermobility syndrome is highly prevalent in general rheumatology clinics, presentation being gender, age and race-related. Ann Rheum Dis 2006; 65 (Suppl 2): 263.

13. Grahame R, Hakim AJ. Hypermobility. Curr Opin Rheumatol 2008; 20: 106-10.

14. Bravo JF, Wolff C, Correa S. Clinical study of 972 rheumatological patients with joint hypermobility syndrome (JHS), using the Brighton criteria. Ann Rheum Dis 2007; 66 (Suppl II): 244 (Abstract).

15. Bravo JF, WolfF C. Clinical study of hereditary disorders of connective tissues in a Chilean population. Joint hypermobility syndrome and vascular
Ehlers-Danlos syndrome. Arthritis Rheum 2006; 54: 515-23.

16. Grahame R. Editorial: Time to take hypermobility seriously (in adults and children). Rheumatology 2001; 40: 485-91.

17. Grahame R, Bird HA. British consultant meumatologists perceptions about the hypermobility syndrome: a national survey. Rheumatology 2001; 40: 560-3.

18. Сніш АH. Joint Hypermobility Syndrome: inherited disorder of collagen synthesis. J Rheumatol 1986; 13: 239-42.

19. Hermanns-Le T, Pierard GE. Ultra structural alterations of elastic fibers and other dermal components in Ehlers-Danlos syndrome of the hypermobile type. Am J Dermatopathol 2007; 29: 370-3.

20. ZweErs MC, Hakim AJ, Grahame R, Schalkwijk J. Joint hypermobility syndromes: the pathophysiologic role of Tenascin-X gene defects [review]. Arthritis Rheum 2004; 50: 2742-9.

21. LevY HP. Ehlers-Danlos Syndrome, Hypermobility Type. http://genetests.org/profiles/eds3/ 22 Oct. 2004.

22. Bravo JF, Wolff C. Clinical study of 1124 cases of Ehlers-Danlos syndrome (Classic EDS, JHS and Vascular EDS). Arthritis Rheum 2007; 56 (Suppl): 1633. (Abstract).

23. BIRD HA, TRIBE CR, BACON PA. Joint hypermobility leading to osteoarthritis and condrocalcinosis. Ann Rheum Dis 1978; 37: 203-11.

24. Rojas C, Aris H, Carvallo A. Esclerosis sistémica progresiva. Análisis clínico de 90 casos. Libro de resúmenes XV Congreso Chileno de Reumatología 1982; pp. 3.

25. Saraux A, Aluain J, Guedes C, Baron D, Youinou P, Le GoFf P. Raynaud's phenomenon in rheumatoid. Arthritis 1996; 35: 752-4.

26. Marice HR, Carpentier PH, Weinrich MC, Keil JE, Franco A, Drouet P et al. Geographic variation in the presence of Raynaud's phenomenon. Charleston, SC, USA vs. Tarentaise, Savoie, France. J Rheumatol 1993; 20: 70-6.

27. Bravo JF, WolfF C. Clinical study of Dysautonomia in 1226 patients with Joint Hypermobility Syndrome. J Clin Rheumatol 2008; 14 (Suppl): S33 (Abstract).

28. Rowe PC, Barron DF, Calkins H, Maumenee T, Tong PY, GERAGHTY MT. Orthostatic intolerance and chronic fatigue syndrome associated with Ehlers-Danlos syndrome. J Pediatr 1999; 135: 494-9.

29. Gazit Y, Nahir AM, Grahame R, Jacob G. Dysautonomia in the joint hypermobility syndrome. Am J Med 2003; 115: 33-40.

30. Arendt-Nielsen L, Kaalund P, Bjerring P, Hogsaa B. Insufficient effect of local analgesics in EhlersDanlos type III patients (connective tissue disorder). Acta Anaesthesiol Scand 1990; 34: 358-61.

31. Maшuk AK, Ferrel Wr, McDonald AG, Sturrock RD. 
Impaired propioceptive acuity at the proximal interphalangeal joint in patients with the hypermobility syndrome. Br J Rheum 1994; 33: 631-7.

32. Gratacos M, Nadal M, Martin-Santos R, Pujana MA, Gago J, Peral B eT aL A polymorphic genomic duplication on human chromosome 15 is a susceptibility factor for panic and phobic disorders. Cell 2001; 106: 367-9.

33. Dolan AL, Arden NK, Grahame R, Spector TD. Assessment of bone in Ehlers-Danlos syndrome by ultrasound and densitometry. Ann Rheum Dis 1998; 57: 630-3.

34. De Paepe A, Devereux RB, Dietz HC, Hennekam RC, PyeRITz RE. Revised diagnostic criteria for the Marfan syndrome. Am J Med Gen 1996; 62: 417-26.

35. BRAVo JF. Importancia de la hipermovilidad articular como causa frecuente de morbilidad, no sólo músculo-esquelética, sino también sistémica: criterios diagnósticos. Reumatología 2003; 19: 33-8.

36. Gedalia A, Press J, Kiein M, Buskila D. Joint hypermobility and fibromyalgia in school children. Ann Rheum Dis 1993; 52: 494-6.

37. Barron DF, Cohen BA, Geraghty MT, Violand R, Rowe PC. Joint hypermobility is more common in children with chronic fatigue syndrome than in healthy controls. J Pediatr 2002; 141: 421-5.
38. Acasuso-Díaz M, Colnantes-Estevez E. Joint hypermobility in patients with fibromyalgia syndrome. Arthritis Care \& Research 1998; 11: 39-42.

39. Engeibert RHH, Bank RA, SakKers RJB, Helders PJM, Beemer FA, Utternaal CSP et al. Pediatric generalized joint hypermobility with and without musculoskeletal complaints: localized or systemic disorder? Pediatr 2003; 111: 248-54.

40. Keer R, Grahame R. Hypermobility Syndrome. Recognition and management for physiotherapists. Edited by Butterworth Heinemann. London. UK. Imprint by Elsevier Science Limited, 2003.

41. McLfan RR, Jacques PF, Selhub J, Tucker KL, Samelson EJ, Broe KE et al. Homocysteine as a predictive factor for hip fracture in older persons. N Engl J Med 2004; 350: 2042-9.

42. Reginster JY, Deroisy R, Rovati LC, Lee RL, Lejeune E, BRUYERE O ET AL. Long term effects of glucosamine sulphate in the progression of osteoarthritis: aleatory clinical study, controlled with placebo. Lancet 2001; 357: 251-6.

43. Leeb BF, Schweitzer H, Montag K, Smolen JS. A metaanalysis of chondroitin sulfate in the treatment of osteoarthritis. J Rheumatol 2000; 27: 205-11. 